\title{
Optimismo, resiliencia, sentido del humor y la salud de personas con pareja
}

Optimism, resilience, sense of humor and health of people with a partner

Otimismo, resiliência, senso de humor e saúde de pessoas com um parceiro

\section{Nicole Flórez ${ }^{1}$ \\ Rozzana Sánchez Aragón ${ }^{2}$}

Recibido: 12 de junio de 2019 Aprobado: 14 de noviembre de 2019 Publicado: 10 de diciembre de 2019

Cómo citar este artículo:

Flórez N. y Sánchez Aragón, R. (2019). Optimismo, Resiliencia, Sentido del Humor y la Salud de Personas con Pareja. Pensando Psicología, 15(26), 1-24. DOI: https://doi.org/10.16925/2382-3984.2019.02.03

Artículo de investigación. https://doi.org/10.16925/2382-3984.2019.02.03

1 Universidad de Nariño

ORCID: https://orcid.org/00000-0002-0298-001X

Correo electrónico: Nicole.florez.r@gmail.com

2 Universidad Nacional Autónoma de México

ORCID: https://orcid.org/0000-0002-5952-8972 


\section{Resumen}

Introducción: Las relaciones de pareja llegan a convertirse en el vínculo más importante para el ser humano ya que en ellas se fomentan y enriquecen ciertos atributos individuales que se evidencian en la interacción romántica. Ejemplo de ello son los constructos de: optimismo, resiliencia y humor positivo -entre otras- que traen consigo contribuciones en el ánimo, la satisfacción con la vida, el bienestar y la salud de las personas.

Objetivo: (1) Identificar el nivel de magnitud en optimismo, resiliencia, sentido del humor y salud, (2) examinar las posibles diferencias según el sexo en dichas variables y (3) conocer el grado de asociación entre el optimismo, la resiliencia y el sentido del humor con la salud de hombres y mujeres con pareja.

Metodología: se realizó un estudio correlacional y comparativo con una muestra no probabilística por conveniencia de 240 personas (50\% mujeres y 50\% hombres) adultos, con edades comprendidas entre los 18 y 75 años, cuya escolaridad mínima fue de preparatoria y tiempo mínimo de relación de seis meses.

Resultados: se destacan la relación positiva que existe entre el optimismo, la resiliencia y el sentido del humor con la salud, especialmente con el factor de vitalidad; y relación negativa con algunos factores que indican problemas con la salud. En cuanto a las diferencias por sexo, se encontró que los hombres presentan mayor confianza en sí mismos, fortaleza, apoyo social, creatividad/ingenio, orientación humorística hacia lo social, prudencia, humor negro y vitalidad en comparación con las mujeres que solamente puntuaron alto en esperanza y pasividad humorística.

Palabras claves: optimismo, resiliencia, relaciones de pareja, salud, sentido del humor.

\section{Abstract}

Introduction: Relationships become the most important link for the human being since they encourage and enrich certain individual attributes that are made evident through romantic interaction. An example of this are the constructs of: optimism, resilience and positive humor - among others - that contribute to the mood, satisfaction with life, well-being and health of people.

Objectives: (1) Identify the levels of optimism, resilience, sense of humor and health; (2) Examine the possible differences according to sex in these variables and; (3) Understand the degree of association between optimism, resilience and sense of humor with the health of men and women with partners.

Methodology: A correlational and comparative study was carried out with a non-probabilistic sample of 240 people for convenience; adults (50\% women and $50 \%$ men), aged between 18 and 75 years old, with a minimum schooling of high school and a minimum relationship time of six months.

Results: The positive relationship between optimism, resilience and sense of humor with health, especially with the vitality factor, is highlighted; along with negative relationships associated with some factors that indicate health problems. As for the differences by sex, men were found to possess greater self-confidence, strength, social support, creativity / ingenuity, social humorous orientation, prudence, dark humor and vitality compared to women, who only scored high on hope and humorous passivity.

Keywords: optimism, resilience, relationships, health, sense of humor.

\section{Resumo}

Introdução: Os relacionamentos se tornam o elo mais importante para o ser humano, pois incentivam e enriquecem certos atributos individuais que são evidenciados por meio da interação romântica. Um exemplo disso são os construtos de: otimismo, resiliência e humor positivo - entre outros - que contribuem para o humor, a satisfação com a vida, o bem-estar e a saúde das pessoas. 
Objetivos: (1) Identificar os níveis de otimismo, resiliência, senso de humor e saúde; (2) Examine as possíveis diferenças de acordo com o sexo nessas variáveis e; (3) Entenda o grau de associação entre otimismo, resiliência e senso de humor com a saúde de homens e mulheres com parceiros.

Metodologia: Foi realizado um estudo correlacional e comparativo com uma amostra não probabilística de 240 pessoas por conveniência; adultos (50\% mulheres e 50\% homens), com idades entre 18 e 75 anos, com escolaridade mínima do ensino médio e tempo mínimo de relacionamento de seis meses.

Resultados: Destaca-se a relação positiva entre otimismo, resiliência e senso de humor com a saúde, principalmente com o fator vitalidade; juntamente com relações negativas associadas a alguns fatores que indicam problemas de saúde. Quanto às diferenças entre os sexos, verificou-se que os homens possuem maior autoconfiança, força, apoio social, criatividade / engenho, orientação social humorística, prudência, humor sombrio e vitalidade em comparação às mulheres, que pontuaram apenas com esperança e passividade humorística.

Palavras-chave: otimismo, resiliência, relacionamentos, saúde, senso de humor.

\section{Introducción}

Una de las relaciones a la que se le otorga mayor importancia en la vida del individuo es la de pareja, puesto que es una conexión sentimental en la cual se busca cercanía, intimidad y apoyo a largo plazo; además de que genera un tipo de compromiso y convivencia amena para alcanzar objetivos conjuntos de las personas que conforman la diada (González, 2017). De igual manera, este vínculo permite establecer un lazo intenso, duradero y familiar, en el cual se experimentan sentimientos de confianza, comprensión, amor, unidad y felicidad en las partes (Sánchez Aragón, García Meraz y De Andrade, 2018). Esta relación fundamentada en el componente emocional, se convierte en una fuente de sensaciones, afectos y sentimientos agradables y satisfactorios en la vida de la persona, los cuales suelen incrementar cada vez que la persona experimenta cercanía con su ser amado (Acevedo, Restrepo de Giraldo y Tovar, 2007).

Cuando la persona evidencia emociones positivas dentro de su relación, se generan respuestas beneficiosas para la persona. Por ejemplo, Alzugaray y García (2015), encontraron que las personas con pareja son más felices que las que no la tienen, muestran un aumento en la flexibilidad para enfrentar los problemas de la vida, la intimidad, la seguridad (Rostami, Taheri, Abdi \& Kermani, 2014), viven un mayor apoyo emocional y protección entre los dos miembros (Varela y Barrón, 2016) y por lo tanto, un incremento en la salud física y mental de los miembros de la parejas (Grinberg, 2017).

En este estudio, la salud es entendida como el funcionamiento adecuado a nivel físico, psicosocial y emocional (Sánchez Aragón, García Meraz y Martínez Trujillo, 2017), es decir, abarca tanto la esfera fisiológica como la afectiva y aquellas 
condiciones que provienen del ambiente social que rodea a la persona (Alcántara, 2008), incluyendo sus relaciones de pareja, las cuales, dependiendo de su calidad, impactarán en su salud física y mental (August, Kelly \& Markey, 2016). En este sentido, se argumenta que la convivencia en pareja se asocia a un mejor perfil de salud con respecto a la población que no la tiene (Gumà, Arpino y Solé-Auró, 2018). Se ha buscado entender de qué manera la interacción con los otros, particularmente la pareja, incrementa el bienestar social y las conductas favorables para la salud (Huneycutt, 2013; Pozos, Rivera Aragón, Reyes y López, 2013).

Con relación en esto, hace algunos años la psicología ha buscado ampliar su foco de estudio, abordando las emociones positivas que contribuyen a la salud y el bienestar de las personas (Ong, Mroczek \& Riffin, 2011). Desde la teoría de ampliación y construcción, se plantea que la experiencia de emociones positivas tiene dos funciones primordiales: ampliar el repertorio de pensamiento acción inmediato de la persona y construir recursos personales que incrementan las posibilidades de afrontamiento y adaptación general del individuo (Barragán y Morales, 2014).

Algunas de las variables de estudio que involucran la experiencia de emociones positivas son: el optimismo, la resiliencia y el humor positivo -entre otras-, mismas que proveen al individuo de recursos para hacer frente y sobreponerse a los momentos difíciles de la vida, resolver problemas y crecer a nivel personal (Park, Peterson \& Sun, 2013). En congruencia, Piqueras, Ramos, Martínez y Oblitas (2009) exponen que al no experimentar este tipo de emociones positivas por largos periodos de tiempo, se pueden producir daños en la salud física y mental como: trastornos cardiovasculares, reumatológicos, inmunológicos, de ansiedad, depresión e ira, entre otros.

El optimismo se refiere a la disposición positiva hacia el futuro y la creencia de que los resultados deseados pueden ser alcanzados, especialmente en situaciones desagradables (Reche, Tutte \& Cortín, 2014). Las personas optimistas asumen expectativas favorables respecto a las dificultades o problemas que se presentan, incrementando sus esfuerzos por obtener un buen resultado (Guzmán, Maragaño, Rojas y Tordoya, 2015). De acuerdo con Reche et al., (2014) el optimismo está compuesto por el control interno y la habilidad para encontrar sentido a las experiencias de la vida, mejorando así la salud mental, la percepción del bienestar, y el incremento de beneficios que contrarresten las enfermedades terminales y crónicas. Asimismo, el optimismo permite modular eventos estresantes, el sufrimiento y las enfermedades graves que surgen en las diferentes etapas de la vida de las personas (Vera Poseck, 2006). Cuando esta variable es disposicional, se encuentra asociada con altos niveles salud física y psicológica, mejor calidad de las relaciones interpersonales, satisfacción con la vida y mayor apoyo social percibido (Guzmán et al., 2015). 
En el ámbito de las relaciones de pareja, el optimismo se encuentra asociado con formas más exitosas de resolver los conflictos dentro de la pareja, lo que podría contribuir o incrementar el bienestar individual y relacional (Guzmán et al., 2015). En otras investigaciones realizadas en población universitaria, se encontró que el optimismo tiene se relaciona fuertemente con el bienestar y el apoyo social, identificando a la pareja como una de las fuentes principales de apoyo. Además, el modo en que las personas se aproximen a la realidad y sus expectativas acerca del futuro, dará resultados favorables en la satisfacción con la vida, promoviendo sentimientos positivos y mejorando el ajuste psicológico del individuo (Marrero y Carballeira, 2010).

Otra variable muy relacionada con la salud es la resiliencia. Este término se define como la capacidad que posee cada individuo de adaptarse, sobreponerse y enfrentarse positivamente a una situación de riesgo y adversidad, generando crecimiento personal e incremento de las competencias con las que actualmente cuenta (Fernandes, Teva y Bermúdez, 2015). Aunado a lo anterior, la resiliencia permite a la persona adaptarse a cambios, enfrentarse a retos y superar situaciones difíciles y estresantes (Reche, Gómez, Martínez y Tutte, 2018),por lo que se podría decir que esta capacidad no se limita a soportar un suceso traumático, sino que implica un resurgir a través de éste; constituyendo con ello un individuo con una nueva dinámica de vida (Vega, Rivera y Quintanilla, 2011).

Cuando una persona es resiliente, es capaz de normalizar su vida aún en condiciones de riesgo, exclusión o experiencias traumáticas (García y Domínguez, 2013) que surgen de su contacto con el ambiente inmediato. En el contexto de pareja, el individuo tiene que lidiar con un sinnúmero de desafíos y retos ante los cuales es necesario ser resiliente para así lograr salir adelante, sentir bienestar y en consecuencia, disfrutar de salud (González, Vázquez y Álvarez, 2013).

De igual modo, en investigaciones sobre resiliencia se ha encontrado que estar en una relación romántica puede ser un factor protector para las personas que enfrentan situaciones de vida catastróficas y desafortunadas, siempre y cuando la relación provea apoyo, sea cálida y represente un recurso emocional significativo, lo que se puede asociar en gran medida con la variable mencionada (Vega et al., 2011). Por ejemplo, la pareja puede involucrarse para ayudar a su compañero a adaptarse y mantener el bienestar durante las situaciones estresantes que atraviese (Sanford, Backer-Fulghum \& Carson, 2016) e incluso emitir conductas más saludables, generando la resiliencia necesaria para afrontar la posibilidad de tener o desarrollar serios problemas de salud (Cabanyes, 2010).

En una investigación realizada por Chok (2000 en González, Valdez, Oudhof van Barneveld y González, 2009) se encontró que personas con características resilientes 
reducían la intensidad del estrés, lo que traía consigo una disminución de signos emocionales negativos como la ansiedad y depresión al tiempo que aumentaba la salud emocional, de manera que la resiliencia no sólo facilita el afrontamiento de adversidades sino también la promoción de la salud mental y emocional.

Finalmente, el sentido del humor es una variable relacionada con la salud que se define como una respuesta emocional provocada por el reconocimiento de un evento o situación divertida, inesperada y sorprendente que se puede dar a causa de una idea, imagen, texto o evento, broma, gesto y/o movimiento (Palomar, Victorio \& Matus, 2011). Cabe resaltar que existen diferentes estilos de humor: (1) el positivo, que produce risa inofensiva, sin tener la intención de ofender o atacar a alguien (Rojas, Peña y Garmendia, 2014) y que busca mejorar las relaciones interpersonales con anécdotas e historias divertidas, comentarios ingeniosos, conductas y reacciones físicas divertidas (Caird y Martin, 2014); y el (2) negro, el cual se usa como un mecanismo que, a través de la ironía y el sarcasmo, permite enfrentar los problemas, liberar el estrés y contribuir al aumento del bienestar físico y psicológico, contrarrestando emociones negativas y trastornos como la depresión y la ansiedad (Ferguson, 2014; Rojas et al., 2014). En conclusión, el humor funciona como una estrategia de afrontamiento que permite deshacerse de tensiones, disipar las preocupaciones y relajar el organismo (Vera Poseck, 2006; Palomar et al., 2011), puesto que, al reír, se liberan endorfinas que permiten la reducción del estrés y la ansiedad, mejorando la calidad de vida, el bienestar emocional y la salud física de un individuo (Ferguson, 2014).

En lo que respecta al papel del sentido del humor en las relaciones de pareja, Bippus, Young y Dunbarhan (2011) documentaron en estudios anteriores que éste contribuye significativamente -como el optimismo y la resiliencia- a la resolución de conflictos y al incremento de las expresiones de afecto y la satisfacción con la relación (Cann, Zapata y Davis, 2011).

De esta manera, en el presente estudio se propuso el abordaje de constructos que, si bien son de gran relevancia para el bienestar y la salud de las personas, no han sido investigados en conjunto en el contexto de la pareja mexicana, aun sabiendo que dichas variables inciden de manera favorable en las personas y pueden fomentar estrategias personales y relacionales que influyan en la manera en la que los individuos se desarrollen dentro del vínculo romántico. Para ello se analizó la relación entre la salud y el optimismo, la resiliencia y el sentido del humor en personas con pareja, a partir de la identificación del nivel de cada variable, el reconocimiento de diferencias entre hombres y mujeres y el grado de asociación entre las variables de trabajo y la salud. 
Con base en lo anterior, los propósitos de esta investigación fueron: (1) Identificar el nivel de magnitud en optimismo, resiliencia, sentido del humor y salud (2) Identificar las diferencias según el sexo en el optimismo, la resiliencia, el sentido del humor y la salud en mujeres y hombres con pareja y (3) Conocer el grado de asociación entre el optimismo, la resiliencia y el sentido del humor con la salud en personas con pareja.

A su vez, las hipótesis del estudio fueron:

H1: Las mujeres en comparación con los hombres reportan mayor optimismo y resiliencia.

H2: Los hombres en comparación a las mujeres reportan niveles más altos en la variable del sentido del humor

H3: Existe una asociación entre el optimismo, la resiliencia y el sentido del humor con la salud

\section{Método}

\section{Participantes}

Se trabajó con una muestra no probabilística por conveniencia (Hernández, Fernández y Baptista, 2006): 240 participantes (120 hombres y 120 mujeres) residentes de la Ciudad de México. Todos adultos de edades entre 18 y 75 años ( $M=35,36$ y $D E=12,152)$ de los cuales 31 tuvieron una escolaridad de preparatoria (12,9\%), 108 licenciatura (45\%) y 101 de posgrado (42\%) al momento del estudio. Con respecto al tiempo de vivir junto a su pareja iba desde 6 meses hasta los 54 años. Los criterios de inclusión a la muestra fueron: (1) ser heterosexuales, (2) mayores de 18 años, (3) tener pareja, y (4) contar con una escolaridad mínima de preparatoria.

\section{Instrumentos}

Con el fin de responder a los propósitos de esta investigación, se aplicaron las siguientes medidas:

Escala de Optimismo (Sánchez Aragón, 2018) en su versión corta, comprende 19 reactivos en formato de respuesta tipo Likert de cinco puntos que indican grados de acuerdo con respecto a cada afirmación donde 1 es "Totalmente en desacuerdo" y 5 "Totalmente de acuerdo". Estos reactivos se distribuyen en cuatro factores: (1) Actitud positiva (5 reactivos y $\alpha=0,90$ ) con ejemplos de reactivos como "Doy la bienvenida a cada nuevo reto" y "Soy optimista aunque parezca que lo que viene va a ser negativo"; (2) Control interno (4 reactivos y $\alpha=0,78$ ) relacionado con ítems como: 
"Para cada problema encuentro una solución" y "Creo que puedo cumplir mis metas"; (3) Autoconfianza (5 reactivos y $\alpha=.81$ ) la cual tiene que ver con reactivos como: "Veo el futuro con confianza" y "Tengo mucha confianza en mí mismo(a)"; y (4) Esperanza (5 reactivos y $\alpha=0,80$ ) con ejemplos de reactivos como: "La mayor parte del tiempo espero que sucedan cosas buenas" y "Creo que mi futuro va a estar muy bien". La validez se obtuvo a través de un análisis factorial de componentes principales y rotación ortogonal, donde los factores explicaron el 55.11\% de la varianza y con un Alpha de Cronbach total de 0,937

Escala de Resiliencia (Palomar Lever y Gómez Valdez, 2010), en su versión corta, comprende 43 reactivos en formato de respuesta tipo Likert de cinco puntos que indican acuerdo con respecto a la afirmación donde 1 es "Totalmente en desacuerdo" y 5 "Totalmente de acuerdo". Estos reactivos se distribuyen en 5 factores que explicaron el 43,60\% de la varianza: (1) Fortaleza y confianza en sí mismo (5 reactivos y $\alpha=0,92)$ con reactivos como: "Soy una persona fuerte" $y$ "Tengo el control de mi vida"; (2) Competencia Social (5 reactivos y $\alpha=0,89$ ) que ejemplo de ítems como: "Es fácil para mí hacer reír a otras personas" y "Me es fácil establecer contacto con nuevas personas"; (3) Apoyo familiar (5 reactivos y $\alpha=0,87$ ) con ejemplos de reactivos como: "Tengo una buena relación con mi familia" y "Aún en momentos difíciles nuestra familia tiene una actitud optimista hacia el futuro"; (4) Apoyo social (5 reactivos y $\alpha=0,84$ ), con ítems como: "Tengo algunos amigos/familia que valoran mis habilidades" y "Siempre tengo alguien que puede ayudarme cuando lo necesito"; y (5) Estructura (5 reactivos y $\alpha=0,79)$ la cual tiene que ver con reactivos como: "Soy bueno(a) para organizar mi tiempo" y "Trabajo mejor cuando tengo metas". La validez se obtuvo a través de un análisis factorial de componentes principales y rotación varimax con un coeficiente Alpha de Cronbach total fue de 0,93.

Escala de Sentido del Humor en la Pareja (Cruz Vargas y Sánchez Aragón, 2018) versión corta. Consta de 24 reactivos en formato de respuesta tipo Likert de cinco puntos que indican grados de acuerdo donde 1 es "Totalmente en desacuerdo" y 5 "Totalmente de acuerdo". Dichos reactivos se distribuyen en cinco factores: (1) Creatividad-Ingenio (5 reactivos y $\alpha=0,79$ ), con ítems como: "Muestro ingenio rápidamente cuando hay que responder a algo" y "A veces invento bromas o historias divertidas"; (2) Orientación humorística y función social (5 reactivos y $\alpha=0,90$ ) con ejemplos de ítems como: "Uso el humor para ganar afecto o aprobación de otros" y "Estoy al día de los nuevos chistes para ganar afecto o aprobación de otros"; (3) Prudencia (5 reactivos y $\alpha=0,79$ ), relacionado con reactivos como: "Tengo un sentido del humor sutil e indirecto" y "A veces hago observaciones humorísticas sin que los demás se den cuenta"; (4) Pasividad humorística (5 reactivos y $\alpha=0,71$ ), con reactivos 
como: "Continuamente fallo en identificar el elemento humorístico de los chistes" y "Disfruto escuchar bromas pero casi no las recuerdo"; y (5) Humor negro (4 reactivos y $\alpha=0,76)$ con ítems como: "Hago parodias que otros podrían interpretarlas como irrespetuosas" y "Soy sarcástico". La validez se obtuvo a través de un análisis factorial que explicaron el $42.16 \%$ de la varianza y un Alpha de Cronbach total de 0,94.

Escala de Salud Física y Psicológica (SF-36) Ilevada a cabo por Zúñiga, Carrillo, Fos, Gandek y Medina (1999) y validada por Sánchez Aragón et al. (2017). Consta de 44 reactivos en dos secciones, la primera con 40 que se encuentran distribuidos en cinco factores que explicaron el 65,36\% de la varianza y que arrojaron coeficientes de confiabilidad Alpha de Cronbach de moderados a moderados altos $(0,76$ a 0,95). Entre las categorías de la primera sección del cuestionario, se encuentran: (a) Rol Emocional y Función Social con ítems como: "¿Qué tanto se sintió desanimado y triste?" y "¿Qué tanto sus problemas emocionales le han dificultado sus actividades sociales (como visitar a los amigos o familiares)?"; (b) Función Física, con ejemplos de reactivos como: "Subir un solo piso por la escalera" y "Hacer esfuerzos moderados, como mover una mesa, pasar la aspiradora, jugar boliche o caminar más de una hora"; (c) Rol Físico, ejemplos de ítems: "¿Tuvo que dejar de hacer algunas tareas en su trabajo o en sus actividades cotidianas, a causa de sus salud física?" y "¿Hizo menos de lo que hubiera querido hacer, a causa de su salud física?"; (d) Dolor Corporal con ejemplos de items: "¿Tuvo dolor en alguna parte del cuerpo?" y "Ha tenido un dolor por largos periodos de tiempo?" y finalmente (e) Vitalidad con reactivos como: "¿Qué tanto se sintió calmado y tranquilo?" y "¿Qué tanto se sintió feliz?". La segunda sección incluyó 4 reactivos en un solo factor que explicó el 55,1\% de la varianza y cuyo coeficiente de confiabilidad fue de 0,73. El factor se nombró Salud General, con reactivos como: "Creo que mi salud va a empeorar" y "Mi salud es excelente".

\section{Procedimiento}

Los instrumentos fueron aplicados por dos estudiantes de psicología en lugares públicos, centrándose especialmente en universidades y centros comerciales, así como también en otros establecimientos donde se pudieran encontrar participantes que cumplieran con los criterios de inclusión. Todos los participantes accedieron de manera libre y voluntaria, garantizando el anonimato y la confidencialidad de sus respuestas. Al presentarse dudas entre los participantes con respecto al cuestionario, se brindó ayuda y se atendieron las interrogantes. 


\section{Análisis de Datos}

Con el fin de responder al primer objetivo, se realizaron análisis descriptivos para identificar la media de cada factor de las escalas aplicadas. Para el segundo objetivo, se realizó una prueba t para muestras independientes y finalmente para el último objetivo, se llevó a cabo análisis de correlación producto-momento de Pearson para examinar la relación entre las variables. Todo lo anterior mediante el uso del programa estadístico SPSS, versión 20.

\section{Consideraciones éticas}

El presente estudio tuvo en cuenta la presentación del consentimiento informado para contar con la colaboración de los participantes. En este documento, se especificó el objetivo y otros aspectos de la investigación, además delas normas éticas establecidas por la American Psychological Association, las cuales cumplieron en su totalidad los investigadores.

\section{Resultados}

Luego de realizar los análisis estadísticos pertinentes para lograr responder a los objetivos planteados, se encontró que en la variable optimismo, los participantes presentan mayor certidumbre hacia el futuro y expectativas favorables en relación a los sucesos que pasarán en su vida (Esperanza); seguridad en cumplir sus objetivos y metas, además de una clara percepción de que son ellos mismos quienes controlan sus vidas (Control interno); presentan una postura positiva en las diferentes situaciones de la vida, percibiendo las situaciones favorables y desfavorables como retos y aprendizajes (Actitud positiva); creen en sí mismos y presentan seguridad en lo que realizan día a día (Autoconfianza). Cabe señalar que todos los datos se encontraron por arriba de la media teórica en la variable optimismo.

En cuanto a la variable resiliencia, se observó que la muestra se caracteriza por percibir alto apoyo por parte de familiares o amigos en situaciones adversas de la vida y de quienes pueden recibir ayuda cada vez que sea necesario (Apoyo social); presentan una buena relación con sus familiares y logran experimentar emociones positivas en los diferentes espacios que comparten con los mismos (Apoyo familiar); son personas que se identifican como fuertes y seguras, capaces de lograr sus planes sin importar los problemas que se presenten en el camino (Fortaleza y confianza en sí mismo); se adaptan muy bien al entorno social y pueden establecer de manera rápida relaciones interpersonales cercanas (Competencia social) y por último, son personas 
que gozan de organización en su vida, lo que les permite adaptarse de mejor manera a los cambios que se presenten (Estructura).

Respecto a la variable sentido del humor, se encontró que estas personas se perciben como creativas a la hora de contar un chiste o inventar una broma, además de que suelen mejorar el impacto humorístico de manera oportuna y rápida (Creatividad/ Ingenio); suelen ser prudentes a la hora de realizar una broma (Prudencia) y en menor medida, realizan chistes ofensivos dirigidos a otras personas que se pueden interpretar como irrespetuosos o groseros (Humor negro). Por otra parte, estas personas presentan ligeras dificultades a la hora de entender un chiste o contar una broma de forma divertida (Pasividad humorística) y se encontraron por debajo de la media en cuanto al uso del humor para ganar la aprobación de los demás o acercarse más a un grupo social (Orientación humorística y función social).

Por último, en la variable Salud, se encontró que los participantes se perciben saludables (Sentirse sano) y tener una buena salud (En general usted diría que su salud es), más o menos igual a la de hace un año (Como diría que es su salud actual, comparada con la de hace un año). Además, se sienten vigorosos, llenos de energía y experimentando emociones positivas (Vitalidad) y en menor medida malestar y dolor (Dolor corporal), sintiéndose propensos a enfermarse en ocasiones futuras y como consecuencia de que su salud decaiga (Riesgo de enfermedad). No presentan inconvenientes en su salud emocional y esto no afecta la manera en que se vinculan con su grupo social cercano (Rol emocional y función social). Asimismo, no evidencian muchas dificultades al ejecutar movimientos motores y físicos en sus actividades diarias (Función física) o afectaciones en la realización de sus tareas o trabajos o la reducción del tiempo que dedican a los mismos (Rol físico) (ver Tabla 1).

Tabla 1. Magnitud de Optimismo, Resiliencia, Sentido del humor y Salud

\begin{tabular}{llll}
\hline \multirow{2}{*}{ Escala } & \multicolumn{1}{c}{ Factor } & Media & DE \\
\hline \multirow{3}{*}{ Optimismo } & Actitud positiva & 3,58 & 0,787 \\
\cline { 2 - 4 } & Control interno & 3,82 & 0,745 \\
\cline { 2 - 4 } & Autoconfianza & 3,27 & 0,856 \\
\cline { 2 - 4 } & Esperanza & 3,92 & 0,725 \\
\hline \multirow{3}{*}{ Resiliencia } & Fortaleza y confianza en sí mismo & 3,75 & 0,603 \\
\cline { 2 - 4 } & Competencia social & 3,66 & 0,739 \\
\cline { 2 - 4 } & Apoyo familiar & 3,93 & 0,709 \\
\cline { 2 - 4 } & Apoyo social & 4,08 & 0,634 \\
\cline { 2 - 4 } & Estructura & 3,61 & 0,741 \\
\hline
\end{tabular}


(viene)

\begin{tabular}{llll}
\hline \multicolumn{1}{c}{ Escala } & \multicolumn{1}{c}{ Factor } & Media & DE \\
\hline \multirow{3}{*}{ Sentido del humor } & \multicolumn{1}{c}{ Creatividad/Ingenio } & 3,62 & 0,775 \\
\cline { 2 - 4 } & Orientación humorística y función social & 2,83 & 0,837 \\
\cline { 2 - 4 } & Prudencia & 3,46 & 0,624 \\
\cline { 2 - 4 } & Pasividad humorística & 2,87 & 0,669 \\
\cline { 2 - 4 } Sumor negro & 3,06 & 1,018 \\
\hline \multirow{3}{*}{ Salud } & Cómo diría que es su salud actual, comparada con la & 3,47 & 0,882 \\
\cline { 2 - 4 } & de hace un año & 3,45 & 0,900 \\
\cline { 2 - 4 } & Función física & 1,35 & 0,657 \\
\cline { 2 - 4 } & Rol físico & 1,25 & 0,546 \\
\cline { 2 - 4 } & Rol emocional y función social & 1,41 & 0,522 \\
\cline { 2 - 4 } & Vitalidad & 3,25 & 0,893 \\
\cline { 2 - 4 } & Dolor corporal & 2,04 & 0,705 \\
\cline { 2 - 4 } & Sentirse sano & 3,61 & 0,962 \\
\cline { 2 - 4 } & Riesgo de enfermedad & 1,93 & 0,929 \\
\hline
\end{tabular}

Fuente: Elaboración propia

En cuanto al segundo objetivo, el cual fue identificar las diferencias estadísticamente significativas entre mujeres y hombres con pareja respecto al optimismo, resiliencia, sentido del humor y salud, se encontró que los hombres presentan más autoconfianza y seguridad en lo que hacen, fortaleza, perciben mayor apoyo y preocupación de su grupo social cercano; utilizan más la creatividad e ingenio para crear bromas e inventar historias divertidas; tienen orientación humorística y función social, lo que les permite ganarse a las personas por medio del humor; prudencia a la hora de contar una broma y no ser ofensivo y humor negro realizando parodias que pueden parecer irrespetuosas, crueles, macabras en comparación con las mujeres. Con respecto a ellas, presentan mayor esperanza hacia el futuro y pasividad humorística a la hora de entender un chiste o broma o ser partícipe de la creación de los mismos (ver Tabla 2). 
Tabla 2. Diferencias según el sexo en optimismo, resiliencia, sentido del humor y salud

\begin{tabular}{|c|c|c|c|c|c|}
\hline Escala & Factor & Mujeres & Hombres & $t$ & $p$ \\
\hline \multirow{2}{*}{ Optimismo } & Autoconfianza & 3,05 & 3,49 & $-4,081$ & 0,000 \\
\hline & Esperanza & 4,06 & 3,78 & 3,061 & 0,002 \\
\hline \multirow{2}{*}{ Resiliencia } & Fortaleza y confianza en sí mismo & 3,67 & 3,83 & $-2,169$ & 0,031 \\
\hline & Apoyo social & 3,96 & 4,20 & $-3,025$ & 0,003 \\
\hline \multirow{5}{*}{ Sentido del humor } & Creatividad/Ingenio & 3,46 & 3,77 & $-3,173$ & 0,002 \\
\hline & $\begin{array}{l}\text { Orientación humorística y función } \\
\text { social }\end{array}$ & 2,61 & 3,04 & $-4,140$ & 0,000 \\
\hline & Prudencia & 3,38 & 3,55 & $-2,181$ & 0,030 \\
\hline & Pasividad humorística & 2,97 & 2,76 & 2,447 & 0,015 \\
\hline & Humor negro & 2,88 & 3,23 & $-2,665$ & 0,008 \\
\hline Salud & Vitalidad & 3,13 & 3,37 & $-2,036$ & 0,043 \\
\hline
\end{tabular}

Fuente: Elaboración propia.

Al analizar el grado de asociación entre el optimismo, la resiliencia y el sentido del humor con la salud, se encontró que cuando las personas se sienten más seguras de sí mismas, confían en sus capacidades (Autoconfianza), establecen fácilmente relaciones sociales y entablan conversaciones sin dificultad alguna (Competencia social), presentan menos problemas con relación en sus emociones y en la convivencia con el grupo de amigos y familiares (Rol emocional y su función social).

Cuando existe la esperanza de cumplir sus metas, la capacidad de encontrar la solución a los problemas (Control interno), mantener la fuerza y la confianza en lo que se hace (Fortaleza y confianza en sí mismo) y presentar la colaboración, además de sentimientos de preocupación y apoyo de sus familiares y amigos (Apoyo familiar y social), se evidencia mayor vigor y energía en su salud (Vitalidad). Al presentar más dificultades para identificar un chiste o crear bromas o historias divertidas, se evidencian más problemas al momento de realizar esfuerzos motores como arrodillarse, correr o bañarse (Función física) y mayor dolor físico (Dolor corporal), lo que genera emociones de tristeza o apatía y afecta sus relaciones sociales como amigos y familiares (Rol emocional y función social).

Cuando los participantes están al día en los chistes y buscan ganarse la aprobación de los demás a través del humor (Orientación humorística y función social), presentan menos afectaciones en sus funciones físicas (Función física). Por el contrario, cuando se evidencia tener un sentido del humor sutil e indirecto (Prudencia), hay menos problemas para realizar tareas domésticas y laborales cotidianas (Rol físico) (ver Tabla 3). 
Tabla 3. Correlaciones entre el optimismo, resiliencia y sentido del humor con la salud

\begin{tabular}{|c|c|c|c|c|c|c|}
\hline \multicolumn{7}{|c|}{ Salud } \\
\hline & Factores & $\begin{array}{l}\text { Función } \\
\text { física }\end{array}$ & $\begin{array}{c}\text { Rol } \\
\text { físico }\end{array}$ & $\begin{array}{l}\text { Rol emocional } \\
\text { y función } \\
\text { social }\end{array}$ & Vitalidad & $\begin{array}{l}\text { Dolor } \\
\text { corporal }\end{array}$ \\
\hline \multirow{2}{*}{ Optimismo } & Control interno & - & - & - & $0,220^{* *}$ & - \\
\hline & Autoconfianza & - & - & $-0,150^{*}$ & $0,176^{* *}$ & - \\
\hline \multirow{4}{*}{ Resiliencia } & $\begin{array}{l}\text { Fortaleza y confianza en } \\
\text { sí mismo }\end{array}$ & - & - & - & $0,241^{\star *}$ & - \\
\hline & Competencia social & - & - & $-0,136^{*}$ & $0,167^{* *}$ & - \\
\hline & Apoyo familiar & - & - & - & $0,135^{\star}$ & - \\
\hline & Apoyo social & - & - & - & $0,174^{\star *}$ & - \\
\hline \multirow{3}{*}{$\begin{array}{l}\text { Sentido del } \\
\text { humor }\end{array}$} & $\begin{array}{l}\text { Orientación humorística } \\
\text { y función social }\end{array}$ & $-0,171^{\star *}$ & - & - & - & - \\
\hline & Prudencia & - & $-0,135^{*}$ & & - & - \\
\hline & Pasividad humorística & $0,173^{\star \star}$ & - & $0,235^{\star \star}$ & - & $0,182^{\star \star}$ \\
\hline
\end{tabular}

${ }^{*} p=0.05{ }^{* *} p=0.01$ Fuente: Elaboración propia

\section{Discusión}

En cuanto a los resultados del primer objetivo, el cual fue identificar el nivel de magnitud en optimismo, resiliencia, sentido del humor y salud, se encontró que los participantes mostraron puntajes altos en el optimismo, es decir; tienen alta esperanza, controlan y se responsabilizan de sus vidas, mantienen una actitud positiva y confianza en sí mismos. Lo anterior se apoya en Peinado (2015) y Guzmán et al. (2015) quienes señalan que estar dentro de una relación de pareja duradera y estable incrementa los niveles de optimismo, una percepción favorable hacia los sucesos de la vida y una actitud más positiva ante el día a día. Además, enfrentar los desafíos junto a la pareja permite generar estrategias de adaptación, aumentando así la probabilidad de obtener más resultados positivos y mejorar la calidad del vínculo (Neff y Geers, 2013). Asimismo, estos resultados son acordes de acuerdo a lo planteado por Turliuc y Candel (2017), quienes plantean que cuando los individuos forman parte de vínculos de pareja más seguros, perciben el futuro con mayor esperanza y, por ende, mayores niveles de satisfacción y optimismo a lo largo de su vida.

En cuanto a la variable de resiliencia, los participantes presentaron alto apoyo social y familiar, proveniente de los amigos, la familia y la pareja; lo cual representa un factor de gran soporte ya que de acuerdo con Diener, Gohm, Suh y Oishi (2000) así como con Sanford et al. (2016), provee de auxilio y protección en momentos de 
adversidad, generando intimidad, apego, confort, cuidado, preocupación, consejo y guía (Vega et al., 2011). De esta forma, se puede lograr el fortalecimiento de los vínculos establecidos a partir de la compresión y la atención que se recibe de dichas fuentes sociales en los momentos negativos, para generar o despertar sentimientos de seguridad y fortaleza a la hora de enfrentar retos y adversidades (Palomar Lever y Gómez Valdez, 2010). Esto se complementa con la autoconfianza y la estructura, las cuales dan al individuo una mayor estabilidad en sus vidas, a pesar de que la adversidad aparezca (Skerrett \& Fergus, 2015).

En términos del Sentido del humor, los participantes mostraron puntajes por encima de la media teórica en creatividad/ingenio, prudencia y humor negro, es decir, inventan bromas, teniendo cuidado de no ofender a su audiencia, pero al mismo tiempo haciendo uso de bromas respecto a ciertos grupos étnicos, enfermedades o connotación sexual. Esto es característico de la población mexicana, como lo expone (Hernández, 2006; Fernández, 2012) que manifiesta que esta cultura se caracteriza por tener buen sentido del humor y crear chistes en diferentes situaciones. Precisamente, esta característica es muy valorada y útil dentro de las relaciones románticas ya que acrecienta sentimientos de cercanía, atracción, felicidad, unión, alivia la tensión y minimiza o evita el conflicto, favoreciendo a la diada (Cruz Vargas y Sánchez Aragón, 2018; Bippus et al., 2011). Ligeramente por debajo de media se encuentra la generación de humor con el propósito de "ganarse" a las otras personas y la pasividad humorística, lo cual resulta hasta cierto punto contradictorio con la búsqueda de congraciarse con el medio social de los mexicanos (Domínguez Espinosa, Aguilera Mijares, Acosta Canales, Navarro Contreras y Ruiz Paniagua, 2012; Orozco, 2014).

En lo que respecta a la salud en general de los participantes, ésta es buena, se sienten vigorosos y llenos de energía y no presentan inconvenientes en su salud emocional. Esto se relaciona con que las personas que se encuentran dentro de una relación romántica, presentan sentimientos de felicidad, tranquilidad, entre otras emociones positivas (Pozos, Rivera, Reidl, Vargas y López, 2013). En general, estudios asociados con el tema exponen que las personas con baja calidad en sus relaciones sociales tienen menos salud física y psicológica (Cazorla, 2014).

Referente al segundo objetivo el cual fue identificar las diferencias según el sexo en el optimismo, la resiliencia, el sentido del humor y la salud en mujeres y hombres con pareja, se encontró que los hombres tienen mayor confianza en sí mismos y en lo que hacen, fortaleza para enfrentar los retos y más vitalidad en comparación con las mujeres. Esto puede estar relacionado con lo que se encuentra regularmente en la literatura, es decir, los hombres socialmente aprenden a soportar y resistir situaciones negativas, pensar que son capaces tener éxito, elegir de manera adecuada 
una alternativa cuando se presente un problema y tener más fuerza, energía, ánimo y vivacidad en comparación con las mujeres (González et al., 2013); estas últimas son socialmente percibidas como dóciles, frágiles, serenas, prudentes, sensibles y colaboradoras dentro de la dinámica familiar (Díaz Loving, 2008). No obstante, un dato interesante es que los hombres perciben mayor apoyo social en comparación con las mujeres, lo cual se encuentra en contradicción con estudios previos ya que se expone que son las mujeres las que perciben más apoyo de su grupo social, puesto que son las mismas las que expresan sus problemas y dificultades a sus amigos, familiares o personas cercanas y por dicha razón, acogen consejos y ayuda de parte sus núcleos cercanos (Moreno y Marrero, 2015). Esto también lo sustentan Prado y Del Águila (2003), quienes encontraron que para el sexo femenino, la utilización de sus habilidades sociales y estrategias más abiertas permite vincularse íntimamente con otras personas y desarrollar redes de apoyo.

En cuanto al sentido del humor, los hombres son más bromistas que las mujeres, aumentando su popularidad en el grupo social; además, sus bromas a veces pueden ser crueles o irrespetuosas ya que es más permitido para ellos a nivel social utilizar un humor fuerte y negro, sin que exista negativa por parte de los demás (Reyes, Lara y Lizcano, 2012). Sin embargo, sí suelen ser prudentes a la hora de usar el humor. Por esta misma razón, las mujeres presentan puntajes más bajos en relación a la hora de contar chistes, hacer bromas o inventar historias divertidas y medias más altas en pasividad humorística.

Para el último objetivo, el cual fue conocer el grado de asociación entre el optimismo, la resiliencia y el sentido del humor con la salud en personas con pareja, se encontró que en la medida en que los(as) participantes tienen sentimientos de confianza en sí mismos(as), entablan conversaciones y se relacionan con otros sin problema, se generan menos afectaciones en la salud emocional y convivencia con amigos y familia. Esto puede ser debido a que el uso adecuado de las habilidades sociales y la interacción continua con el grupo social cercano disminuyen afectos negativos en el individuo (Vega et al., 2011). Asimismo, tener sentimientos de confianza y seguridad permiten relacionarse de mejor manera con los que le rodean y sentirse mejor consigo mismo(a).

Por otra parte, se logró demostrar que las personas que tienen la esperanza de cumplir sus metas, creer en sí mismos, ser fuertes cuando existen dificultades y percibir apoyo de sus familiares y amigos, presentan más vigor y energía en su salud y las actividades que ejecutan, lo que puede verse relacionado con el hecho que las personas que experimentan emociones positivas generan mayor actividad física, energía y eficacia con respecto a las funciones vitales (Vecina, 2006; Panish, 2002). 
En congruencia, González et al. (2013) sustentan que una perspectiva positiva de las cosas no solamente alimenta la capacidad para sobreponerse a las desgracias, sino que también vigoriza y fortalece diferentes aspectos del individuo como por ejemplo funciones ejecutivas, motoras y estado de ánimo.

En otro orden de ideas, también se observó que al presentar más dificultades para identificar un chiste o crear bromas o historias divertidas, se evidencian más problemas al nivel motor y dolor físico, lo cual impide o interrumpe las labores, genera emociones de tristeza o apatía y afecta sus relaciones sociales como amigos y familia; esto se sustenta a partir de Palomar et al. (2010) y Vázquez y Castilla (2007) quienes exponen que las personas rígidas y carentes de sentido del humor, suelen exteriorizar mayores dolencias e incrementan la posibilidad de presentar algún trastorno como depresión, ansiedad o deterioro general en la salud. Al mismo tiempo, DeKeyser \& Jacobs (2014) manifiestan que los individuos que no tienen un gran sentido del humor pueden ser menos competentes y atractivos socialmente, trayendo como resultado niveles bajos de satisfacción en las relaciones sociales.

Por el contrario, cuando los participantes están al día en los chistes y buscan ganarse la aprobación de los demás a través del humor junto con un sentido del humor más sutil, presentan menos afectaciones en sus funciones físicas asociadas con realizar tareas domésticas y labores cotidianas. Este hallazgo se confirma con lo que plantean Palomar et al. (2010), donde las personas que gozan de buen humor, suelen involucrarse en actividades más saludables, lo que favorece a su salud física y las funciones que realizan. Asimismo, el humor sirve para liberar tensiones, disipar las preocupaciones y relajar el organismo (Vera Poseck, 2006; Alpízar y Salas, 2010). Empero, es necesario mencionar que no se encontraron relaciones en todas las dimensiones de salud que evalúa el instrumento; esto puede deberse a que el optimismo, la resiliencia y el sentido del humor no tiene que ver con las funciones físicas y motoras que permite el funcionamiento diario del individuo como caminar, correr, realizar labores del hogar o tareas en el sitio de trabajo; sino, más que nada, con la percepción de salud y energía que la persona construye de sí mismo(a) y la manera en la que lo(a) hace sentir y relacionarse con otros.

Finalmente, la presente investigación pudo cumplir con los tres objetivos planteados, encontrando correlaciones bajas pero significativas en las variables de optimismo, resiliencia y sentido del humor con salud, así como también, diferencias interesantes entre hombres y mujeres; lo que contribuirá de manera relevante a entender la utilización de estas variables en el contexto romántico y su implicación en la salud de las personas. De igual forma, se lograron identificar vacíos de conocimiento, especialmente en la variable de sentido del humor, puesto que no se encuentra 
suficiente información referente a esta variable en población mexicana, contribuyendo este estudio a apoyar o complementar la teoría expuesta hasta el momento. En cuanto a las limitaciones del estudio, se reconoce las bajas asociaciones entre las variables, además de la falta de comparación con una muestra que no se encuentre dentro de una relación romántica (personas sin pareja), lo cual fundamentaría de mejor manera que el optimismo, la resiliencia y el sentido del humor incrementan y tienen una fuerte incidencia en las relaciones de pareja.

Se sugiere que para futuras investigaciones, se indague y conceptualice a profundidad el sentido del humor en población mexicana y las diferencias con otras culturas; y se estudie el efecto directo de dichas variables en la salud, para así, instaurar intervenciones que incrementen y fomenten el optimismo, la resiliencia, el sentido del humor y la salud de las personas con pareja.

\section{Referencias}

Acevedo, V., Restrepo de Giraldo, L. y Tovar, J. (2007). Parejas satisfechas de larga duración en la ciudad de Cali. Pensamiento Psicológico, 3(8), 85-107. Recuperado de http://www.redalyc. org/html/801/80130808/

Alcántara, G. (2008). La definición de salud de la Organización Mundial de la Salud y la interdisciplinariedad. Sapiens. Revista Universitaria de Investigación, 9(1), 93-107. Recuperado de http:// www.redalyc.org/articulo.oa?id=41011135004

Alpízar, H. y Salas, D. (2010). El papel de las emociones positivas en el desarrollo de la Psicología Positiva. Revista electrónica de estudiantes. Escuela de psicología, 5(1), 65-83. Recuperado de https://dialnet.unirioja.es/servlet/articulo?codigo=3922019

Alzugaray, C. y García, F. (2015) Relaciones de Pareja y Bienestar. En M. Bilbao, D. Páez y J. C. Oyanedel (Eds.), La felicidad de los chilenos: Estudios sobre bienestar (pp.237-251). Santiago de Chile: RIL editores.

August, K., Kelly, C. \& Markey, C. (2016). Marriage, Romantic Relationships, and Health. Encyclopedia of Mental Health, 3(1), 46-52. doi:10.1016/B978-0-12-397045-9.00074-4

Barragán, A. y Morales, C. (2014). Psicología de las emociones positivas: generalidades y beneficios. Enseñanza e Investigación en Psicología, 19(1), 103-118. Recuperado de https://www.redalyc. org/pdf/292/29232614006.pdf 
Bippus, M., Young, S. \& Dunbar, N. (2011). Humor in conflict discussions: Comparing partners' perceptions. Humor. International Journal of Humor Research, 24(3), 287-303. doi: 10.1515/ humr.2011.018

Cabanyes, J. (2010). Resiliencia: una aproximación al concepto. Revista de Psiquiatría y Salud Mental, 3(4), 145-151.doi: 10.1016/j.rpsm.2010.09.003

Caird, S. \& Martin, R. A. (2014). Relationship-focused humor styles and relationship satisfaction in dating couples: A repeated-measures design. Humor, 27(2), 227-247. doi: 10.1515/ humor-2014-0015

Cann, A., Zapata, C. \& Davis, H. B. (2011). Humor style and relationship satisfaction in dating couples: Perceived versus self-reported humor styles as predictors of satisfaction. Humor. International Journal of Humor Research, 24(1), 1-20. doi: 10.1515/humr.2011.001

Cazorla, J. (2014). Las relaciones sociales en salud mental: estudio sobre la percepción del impacto de las relaciones sociales en la mejora de la autonomía de las personas con diversidad mental y la relación con el trabajo social. Alternativas: Cuadernos de Trabajo Social, 21(8),161-176. Recuperado de https://rua.ua.es/dspace/bitstream/10045/43716/6/Alternativas_21_08.pdf

Cruz Vargas, D. y Sánchez Aragón, R. (2018). Sentido del humor en la pareja: diferencias por etapa y sexo. La psicología social en México. Volumen XVII, México D. F.: D.R. Asociación Mexicana de Psicología Social A.C.

DeKeyser, F. \& Jacobs, J. (2014). The effect of humor on elder mental and physical health. Geriatric Nursing, 35(3), 205-211. https://doi.org/10.1016/j.gerinurse.2014.01.005

Díaz Loving (2008). Etnopsicología Mexicana: siguiendo la huella teórica yempírica de Díaz-Guerrero, México D. F., México: Trillas

Diener, E., Gohm, C. L., Suh, E. \& Oishi, S. (2000). Similarity of the relations between marital status and subjective well-being across cultures. Journal of Cross-Cultural Psychology, 31(1) 419 436. doi:10.1177/0022022100031004001

Domínguez Espinosa, A. C., Aguilera Mijares, S., Acosta Canales, T., Navarro Contreras, G. y Ruiz Paniagua, Z. (2012). La deseabilidad social revalorada: más que una distorsión, una necesidad de aprobación social. Acta de Investigación Psicológica, 2(3), 808-824. Recuperado de http://www.scielo.org.mx/scielo.php?script=sci_arttext\&pid=S2007-48322012000300005 
Ferguson, D. (2014). Is Laughter the Best Medicine? An Examination of Couples' Abilities to Cope with Cancer by using Humor (tesis doctoral). The Chicago School of Professional Psychology, Chicago.

Fernandes, L., Teva, I. y Bermúdez, M. (2015). Resiliencia en adultos: una revisión teórica. Terapia Psicológica, 33(3), 257-276. Recuperado de https://scielo.conicyt.cl/scielo. php?pid=S0718-48082015000300009\&script=sci_arttext\&tlng=en

Fernández, I. (2012). Los mexicanos somos... El chiste como autorepresentación y estereotipo del mexicano. Diacronie. Studi di Storia Contemporanea, 11(3), 1-19. doi:10.4000/diacronie.2700

García, M. y Domínguez, E. (2013). Desarrollo teórico de la Resiliencia y su aplicación en situaciones adversas: Una revisión analítica. Revista Latinoamericana de Ciencias Sociales, Niñez y Juventud, 11(1), 63-77. Recuperado de https://www.redalyc.org/pdf/773/77325885001.pdf

González, I. (2017). Factores que influyen en la elección y estabilidad en la pareja (tesis de licenciatura). Universidad Nacional Autónoma de México, Facultad de México, México.

González, N., Valdez, J., Oudhof van Barneveld, H. y González, S. (2009). Resiliencia y salud en niños y adolescentes. Ciencia ergo sum, 16(3), 247-253. Recuperado de http://www.redalyc.org/ html/104/10412057004/

González, M., Vázquez, O. y Álvarez P. (2013) El análisis de la resiliencia en personas que constituyen parejas mixtas en Andalucía. Cuadernos de Trabajo Social, 26(2), 275-284. doi: 10.5209/ rev_CUTS.2013.v26.n2.41474

Grinberg, A. (2017). Implementation of brief preventative couples intervention in a primary care setting (tesis doctoral). The University of Arizona, Phoenix.

Gumà, J., Arpino, B. y Solé-Auró, A. (2018). Determinantes sociales de la salud de distintos niveles por género: educación y hogar en España. Gaceta Sanitaria, 33(2), 127-133. doi: 10.1016/j. gaceta.2017.11.010

Guzmán, M., Maragaño, N., Rojas, M. y Tordoya, P. (2015). Apego romántico y optimismo en adultos chilenos. Salud \& Sociedad, 6(3), 224-235. Recuperado de http://www.redalyc.org/ html/4397/439744554003/

Hernández, R., Fernández, C. y Baptista, P. (2006). Metodología de la investigación, México D. F: McGraw-Hill Interamericana Editores. 
Hernández, V. (2006). Antología del Albur Mexicano. Charleston SC, USA: BooksSurge Publishing.

Huneycutt, A. (2013). Altruistic action and relationship satisfaction: Exploring possible benefits of volunteering and altruism on couple relationships (tesis doctoral). Alliant International University, California School of Professional Psychology, Sacramento.

Marrero, R. y Carballeira, M. (2010). El papel del optimismo y del apoyo social en el bienestar subjetivo. Salud Mental, 33(1), 39-46. Recuperado de http://www.redalyc.org/pdf/582/58215 621005.pdf

Moreno, Y. y Marrero, R. (2015). Optimismo y autoestima como predictores de bienestar personal: diferencias de género. Revista Mexicana de Psicología, 32(1), 27-36. Recuperado de http:// www.redalyc.org/articulo.oa?id=243045363004

Neff, L. A. \& Geers, A. L. (2013). Optimistic expectations in early marriage: A resource or vulnerability for adaptive relationship functioning?. Journal of Personality and Social Psychology, 105(1), 38-60. doi: 10.1037/a0032600

Ong, A., Mroczek, D. \& Riffin, C. (2011). The Health Significance of Positive Emotions in Adulthood and Later Life. Social and Personality Psychology Compass, 5(8), 538-551. doi:10.1111/j.1751-9004.2011.00370.x

Orozco, C. (2014). La deseabilidad social en mexicanos religiosos y con autocontrol, una necesidad de aprobación social (tesis de licenciatura). Universidad Nacional Autónoma de México, Facultad de Psicología, México.

Palomar, J., Victorio, A. y Matus, G. (2011) Sentido del humor y optimismo: Un estudio de validación. Interamerican Journal of Psychology, 45(2), 123-132. Recuperado de http://www.redalyc.org/html/284/28422741003/

Palomar Lever, J. y Gómez Valdez, N. (2010). Desarrollo de una escala de medición de la resiliencia con mexicanos (RESI-M). Interdisciplinaria, 27(1), 7-22. Recuperado de http://www.redalyc. org/pdf/180/18014748002.pdf

Panish, J. (2002). Life satisfaction in the elderly: The role of sexuality, sense of humor, and health. Dissertation Abstracts International, 63(5-B).

Park, N., Peterson, C. \& Sun, J. (2013). La Psicología Positiva: Investigación y aplicaciones. Terapia psicológica, 31(1), 11-19. Recuperado de https://scielo.conicyt.cl/pdf/terpsicol/v31n1/ art02.pdf 
Peinado, A. I. (2015). Inteligencia Emocional y Optimismo en pacientes con cáncer y sus parejas: Relación con la calidad de vida de la persona enferma, calidad de la relación, nivel de sobrecarga en la pareja y diferencias de género (tesis doctoral). Universidad de Murcia, España.

Piqueras, J., Ramos, V., Martínez, A. y Oblitas, L. (2009). Emociones negativas y su impacto en la salud mental y física. Suma Psicológica, 16(2), 85-112. Recuperado de http://www.redalyc. org/articulo.oa?id=134213131007

Pozos, J., Rivera Aragón, S., Reyes, I. y López, M. (2013). Escala de Felicidad en la Pareja: Desarrollo y Validación. Acta de Investigación Psicológica, 3(3), 1280 - 1297. doi: 10.1016/ S2007-4719(13)70967-0

Pozos, J., Rivera Aragón, S., Reidl, L., Vargas, B. y López, M. (2013). Felicidad general y felicidad en la pareja: diferencias por sexo y estado civil. Enseñanza e Investigación en Psicología, 18(1), 69-84. Recuperado de https://www.redalyc.org/articulo.oa?id=29228948005

Prado, R. y Del Águila, M. (2003). Diferencia en la resiliencia según género y nivel socioeconómico en adolescentes. Persona, 6(1), 179-196. Recuperado de http://www.redalyc.org/articulo. oa?id=147118110009

Reche, C., Gómez, M., Martínez, A. y Tutte, V. (2018). El optimismo como contribución a la resiliencia deportiva. Revista Iberoamericana de Psicología del Ejercicio y el Deporte, 13(1), 131-136. Recuperado de http://www.redalyc.org/pdf/3111/311153534013.pdf

Reche, C., Tutte, V. y Cortín, F. (2014). Resiliencia, optimismo y burnout en Judokas de competición uruguayos. Revista Iberoamericana de Psicología del Ejercicio y el Deporte, 9(2), 267-279. Recuperado de https://dialnet.unirioja.es/descarga/articulo/4941480.pdf

Reyes, M., Lara, G. y Lizcano, E. (2013). Apreciación del sentido del humor en estudiantes universitarios. Avances en Psicología Latinoamericana, 29(2), 344-353. Recuperado de http://www. scielo.org.co/pdf/apl/v29n2/v29n2a13.pdf

Rojas, A., Peña, N. y Garmendia, C. (2014). Estilos de humor según género, edad y tipo de institución educativa en adolescentes. Eureka: Asunción, 11(1), 20-36. Recuperado de http://docs. bvsalud.org/biblioref/2018/06/905592/eureka-11-1-11.pdf

Rostami, M., Taheri, A., Abdi, M. \& Kermani, N. (2014). The Effectiveness of Instructing Emotionfocused Approach in Improving The marital Satisfaction in Couples. Procedia - Social and Behavioral Sciences, 114(1), 693-698. doi:10.1016/j.sbspro.2013.12.769 
Sánchez Aragón, R., García Meraz, M. y Martínez Trujillo, B. (2017). Encuesta de Salud SF-36: Validación en Tres Contextos Culturales de México. Revista Iberoamericana de Diagnóstico y Evaluación - e Avaliação Psicológica. RIDEP, 45(3), 5-16. doi:10.21865/RIDEP45.3.01

Sánchez Aragón, R., García Meraz, M. y De Andrade, A. (2018). Tácticas de resolución de conflicto en las relaciones de pareja: validación en dos ciudades de México y una de Brasil. Revista Mexicana de Investigación en Psicología, 10(1), 51-62. Recuperado de https://www.medigraphic.com/pdfs/revmexinvpsi/mip-2018/mip181e.pdf

Sánchez Aragón, R. (2018). El inicio y el final de la pareja: variaciones en admiración, optimismo y pasión romántica. En R. Díaz Loving, L. Reyes Lagunes y F. López Rosales (Eds.), La psicología social en México. Volumen XVII (p.999). México D. F.: D.R. Asociación Mexicana de Psicología Social A.C.

Sanford, K., Backer-Fulghum, L. \& Carson, C. (2016). Couple Resilience Inventory: Two Dimensions of Naturally Occurring Relationship Behavior During Stressful Life Events. Psychological Assessment, 28(10), 1243-1254. Recuperado de http://psycnet.apa.org/ doiLanding?doi=10.1037\%2Fpas0000256

Skerrett, K.\&Fergus, K. (Eds.). (2015). Coupleresilience:Emerging perspectives. SpringerNetherlands.

Turliuc, M.\&Candel, O. (2017). Ideal Standards, Optimism and Couple Satisfaction among Romanian couples: Actor, Partner and Similarity Effects. Psihologia Socială, 40(2), 83-96. Recuperado de https://www.researchgate.net/publication/322869895_Ideal_Standards_Optimism_and_ Couple_Satisfaction_among_Romanian_couples_Actor_Partner_and_Similarity_Effects

Varela, I. y Barrón, A. (2016). La influencia de la pareja en el ajuste a la enfermedad cardiaca. Acta de Investigación Psicológica, 6(2), 2459-2468. doi: 10.1016/j.aipprr.2016.06.009

Vázquez, C. y Castilla, C. (2007). Emociones positivas y crecimiento postraumático en el cáncer de mama. Psicooncología, 4(2-3), 385-404. Recuperado de http://revistas.ucm.es/index.php/ PSIC/article/view/PSIC0707220385A/15628

Vecina, M. (2006). Emociones positivas. Papeles del Psicólogo, 27(1), 9-17. Recuperado de http:// www.redalyc.org/articulo.oa?id=77827103

Vega, M., Rivera, M. y Quintanilla, R. (2011). Recursos psicológicos y resiliencia en niños de 6, 8 y 10 años de edad. Revista de Educación y Desarrollo, 17(2), 33-41. Recuperado de http://www. cucs.udg.mx/revistas/edu_desarrollo/anteriores/17/017_Vega.pdf 
24 Optimismo, resiliencia, sentido del humor y la salud de personas con pareja

Vera Poseck, B. (2006). Psicología positiva: una nueva forma de entender la psicología. Papeles del Psicólogo, 27(1), 3-8. Recuperado de http://www.redalyc.org/html/778/77827102/

Zuñiga, M., Carrillo, G., Fos, P., Gandek, B. y Medina, M. (1999). Evaluación del estado de salud con la encuesta SF-36: resultados preliminares en México. Salud Pública de México, 41, 110-118. Recuperado de https://www.redalyc.org 